\title{
COMENTARIOS A UN INFORME DEL PROFESOR GEORGES MARIDAKIS ACERCA DEL REENVÍO*
}

\author{
COMMENTS ON PROFESSOR GEORGES MARIDAKIS' \\ REPORT ON RENVOI
}

Hans KELSEN

Resumen:

Traducción de una carta que el Profesor Hans Kelsen envió al Profesor Maridakis, de la Universidad de Atenas, en la que hace algunas observaciones a un proyecto de discurso a presentar en la Conferencia de La Haya. En esta carta y a partir de su Teoría Pura del Derecho, Kelsen hace algunas observaciones en relación con la noción de reenvío en el Derecho Internacional Privado. Para Kelsen el llamado reenvío es una cuestión de política legislativa y cada estado es libre de admitirlo o no; por lo tanto, en modo alguno es necesario que el legislador deba incorporarlo.

\section{Palabras clave:}

Teoría pura del derecho, norma jurídica, justicia, Derecho Internacional Privado, incorporación del derecho extranjero.

\footnotetext{
* Artículo recibido el 7 de julio de 2013 y aceptado para su publicación el 20 de agosto de 2013.

Traducción de Jorge Alberto Silva, Presidente de la Asociación Mexicana de Profesores de Derecho Internacional Privado. Investigador nacional CONACYT, México, silvasi@aol.com Artículo publicado originalmente en francés como: Observations d'Hans Kelsen, Annuaire de Droit International, Quarante Septième Volume, Session d'Amsterdam, Septembre 1957, tome II, pages 115 à 125, Editions A. Pedone. La traducción al español de este artículo fue autorizada por Editions A. Pedone y el Anuario de Derecho Internacional.
} 
Esta revista forma parte del acervo de la Biblioteca Jurídica Virtual del Instituto de Investigaciones Jurídicas de la UNAM

\section{HANS KELSEN}

Abstract:

Translation of a letter from Professor Hans Kelsen to Professor Maridakis from the University of Athens. In this letter Kelsen formulates comments on Maridakis' draft speech regarding the notion in Private International Law of reenvoi, speech presented at the Hague Conference. Kelsen's comments arise from his Pure Theory of Law and argues that the notion of reenvoi is a matter of legislative policy where each state is free to admit or reject it, therefore for Kelsen it is not necessary for a legislature to adopt reenvoi regulations.

Keywords:

Pure Theory of Law, Legal Norm, Justice, Private International Law, Incorporation of Foreign Law. 
Esta revista forma parte del acervo de la Biblioteca Jurídica Virtual del Instituto de Investigaciones Jurídicas de la UNAM www.juridicas.unam.mx

http://biblio.juridicas.unam.mx

COMENTARIOS AL PROFESOR G. MARIDAKIS

SUMARIO: I. Presentación del traductor. II. Observaciones de Hans Kelsen al informe del profesor Georges Maridakis. III. Bibliografia.

\section{PRESEnTACión DEL TRADUCTOR}

En recuerdo a la joven doctora Sonia Rodriguez, imagen de candor, bonhomía y alegría por la vida. Mi agradecimiento por la amistad que me brindó. iSe fue antes que nosotros! Fue una desgracia

Hace tiempo publiqué un estudio en el que me referí a la teoria de la incorporación (propia del Derecho Internacional Privado), que en gran medida acogí. Durante mi trabajo de investigación encontré un párrafo de Hans Kelsen, aunque no su estudio completo, sino sólo un pequeño párrafo de su exposición en el que abordaba un punto sobre la incorporación.

Dicho apartado lo encontré en un curso que impartió el profesor Edoardo Vitta en La Haya. ${ }^{1}$ Vitta insertó en unas lineas (redactadas en lengua francesa) que corresponden al párrafo de una carta signada por Kelsen, aunque no el documento completo. Las líneas reproducidas por el profesor Vitta me parecieron de importancia para el trabajo que yo realizaba, por lo que hice todo cuanto estuvo a mi alcance para obtener el texto completo de Kelsen, sin éxito alguno. Incluso, le pedí el favor a la Lic. Mayela Celis, collaboratrice juridique, en la Conférence de La Haye de Droit International Privé, para que me auxiliara a su localización en la Bibliothèque du Palais de la Paix (Países Bajos). Mayela solo obtuvo una copia de la obra completa de Vitta (de su curso), que luego me envió. Con este dato y otros que ya poseía armé mi estudio, mismo que hoy se encuentra publicado. ${ }^{2}$

1 Vitta, Edoardo, Cours générale de droit internationale prive, vol. 126, 1979-1, pp. 32 y 33.

2 Silva, Jorge Alberto, Aplicación de normas conflictuales. La aportación del juez, México, UACJ-Fontamara. 2010, aparece en el capítulo 4, p. 109. 
Esta revista forma parte del acervo de la Biblioteca Jurídica Virtual del Instituto de Investigaciones Jurídicas de la UNAM www.juridicas.unam.mx

http://biblio.juridicas.unam.mx

HANS KELSEN

En el mes de abril de 2013 asistí a la Universidad Complutense de Madrid al VII Seminario de Derecho Internacional Privado. Charlando con el Profesor Sixto Sánchez Lorenzo, catedrático de DIPr en la Universidad de Granada, salió a la plática la citada carta de Kelsen. Sixto me dijo que poseía una copia del documento completo, por lo que le rogué me auxiliase a obtener una para mí. Ya de regreso a casa, Sixto me envió por correo una copia del documento que le solicité. La copia del documento que recibí se publicó en el Annuaire de l'Institut de Droit International, en 1957.3 El profesor Sánchez Lorenzo me obsequió, además, un estudio de su autoría en el que hace una cita a esta carta de Kelsen. ${ }^{4}$ Aprovecho la oportunidad para expresar una vez más mi agradecimiento al Profesor Sánchez Lorenzo por la copia y su estudio.

El documento de Kelsen aparece en el citado Annuaire con el título "13. Observations de M. Hans Kelsen". La publicación transcribe la carta sin referencia alguna al motivo o razón del porqué la escribió. Digo lo anterior, ya que presentándose como si fuese una carta, no aparece anotado a quién se la dirigió. Kelsen solo se refiere a su "querido colega".

Cabe agregar que este documento no es fácil obtenerlo y es prácticamente desconocido. En su momento lo busqué entre mis amigos y conocidos, filósofos y teóricos del derecho, sin éxito alguno. 5

Con los elementos que poseo puedo complementar algunos datos sobre la historia y razón de este documento de

3 Annuaire de l'Institut de Droit International, Quarante-septième, Session d'Amsterdam, Bale, Editions juridiques et sociologiques, t. II, septiembre de 1957. El texto del documento de Kelsen aparece a partir de las páginas 115 y ss.

4 Sánchez Lorenzo, Sixto, "Estado democrático, posmodernismo y el derecho internacional privado", Revista de Estudios Juridicos, segunda época núm. 10, Universidad de Jaen, 2010.

5 En realidad, durante la búsqueda que hice del documento nunca pensé que pudiera estar en un Annuaire y en lengua francesa. No busqué por ahí.

PROBLEMA

Anuario de Filosofia y Teoría del Derecho, Núm. 8, enero-diciembre de 2014, pp. 431-448 
Esta revista forma parte del acervo de la Biblioteca Jurídica Virtual del Instituto de Investigaciones Jurídicas de la UNAM www.juridicas.unam.mx

http://biblio.juridicas.unam.mx

COMENTARIOS AL PROFESOR G. MARIDAKIS

Kelsen. Se trata de una carta que le envió el Profesor Kelsen al profesor Georges Maridakis, de la Universidad de Atenas ${ }^{6}$ en respuesta a algunas interrogantes, cuyo contenido literal desconozco.

En 1957 el Profesor Maridakis presentó en la Academia de La Haya un informe relacionado con "el reenvío en el DIPr". El proyecto de este informe fue el documento en torno al cual giró el estudio de Kelsen, que aquí presento. Maridakis lo tituló como "Le renvoi en droit international privé rapports provisoire, définit et supplémentaire" (el reenvío en el derecho internacional privado informes provisional, definitivo y suplementario). ${ }^{7}$

El Profesor Hans Kelsen es sobradamente conocido, lo que me permite omitir su presentación. No obstante, solo haré una referencia brevísima a algunas de sus incursiones en el derecho internacional, generalmente el internacional público y reducidamente el internacional privado. Lo anterior, para ubicar en contexto la carta que aquí presento.

En los inicios de su vida destaca su obra El problema de la soberanía y la teoría del derecho internacional, de 1920. Cuando estuvo en Ginebra ocupó la cátedra de derecho internacional en el Institut Universitaire des Hautes Etudes Internationales, donde se mantuvo hasta 1940, a pesar de la Segunda Guerra Mundial. Unos seis años antes había dado a conocer su Teoría pura del derecho (primera versión).

6 Maridakis (1890-1979) fue rector de la Universidad de Atenas, obtuvo su doctorado en derecho en 1911 en la Escuela de Derecho de Atenas, donde fue profesor de DIPr a partir de 1925; posteriormente Ministro de Justicia en 1952 y en 1954; luego, enseñó en la Academia Internacional de La Haya. Fue miembro del comité de redacción del código civil griego entre 1952 a 1958. Además, fue presidente del comité que redactó el Greek Code of Private Shipping Law. Fue también el primer juez griego en la Corte Europea de Derechos Humanos, durante 11 años. En su currículo cuenta que también fue miembro de la Corte Permanente de La Haya.

7 23a. Commission, Institut de Droit International, Annuaire 17, 1957. 
Esta revista forma parte del acervo de la Biblioteca Jurídica Virtual del Instituto de Investigaciones Jurídicas de la UNAM www.juridicas.unam.mx

http://biblio.juridicas.unam.mx

HANS KELSEN

Debido a la Segunda Guerra mundial emigró a EUA; primero, a la Universidad de Harvard, donde publicó Law and Peace in International Law (1940-41), luego a la Universidad de California (Berkeley), donde fue designado (1945) para impartir International Law, Jurisprudence, and Origin of Legal Institutions. En este mismo año publicó su Teoría general del derecho y el estado. ${ }^{8}$ Poco después (en 1950) publicó The Law of the United Nations. A Critical Analysis of its Fundamental Problems, así como su Teoría pura del derecho, aunque esta última en lengua francesa. ${ }^{9}$ En 1957 publicó la carta dirigida al profesor Maridakis (cuya traducción aquí reproduzco). Más tarde, en 1951, la UNAM le otorgó el grado de Doctor honoris causa y, en 1952, publicó Principles of International Law.

Kelsen se interesó en el informe provisional del profesor Maridakis y en torno al mismo (el rapport provisoire) redactó la carta que aquí presento, respetando el título con el que fue publicada en el Annuaire de l'Institut de Droit International, bajo el título Observaciones de Hans Kelsen, aunque yo le agregaré "al informe del profesor Georges Maridakis".

Debo resaltar que el documento que Kelsen leyó no fue precisamente el informe final, publicado posteriormente por la Conferencia de La Haya (el rapport définit), ${ }^{10}$ según se entiende de la única nota al pie de página puesta por Kelsen. Más bien, dictaminó sobre el informe provisional, que seguramente Maridakis sometió a su consideración (lo que Maridakis llama rapport provisoire). En una nota al pie de

8 Traducida al castellano por Eduardo García Máynez, y publicada en México por la Imprenta Universitaria, en 1950.

9 Una traducción al español se publicó por el Instituto de Investigaciones Jurídicas (UNAM) en 1982.

10 El documento final se publicó como Le renvoi en droit international privé, Rapport définitif, en el Annuarie de L'Institut de droit international (session d'Amsterdam), vol. 4, II, (1957). Así como por la Conferencia de La Haya como Le renvoi en droit international privé (procès-verbaux de la discussion du Rapport de M. Maridakis), en el Annuaire de l'Institut de droit international, vol. 49, tome II, Session de Salzbourg, 1961. 
Esta revista forma parte del acervo de la Biblioteca Jurídica Virtual del Instituto de Investigaciones Jurídicas de la UNAM www.juridicas.unam.mx

http://biblio.juridicas.unam.mx

COMENTARIOS AL PROFESOR G. MARIDAKIS

página, Kelsen autorizó a Maridakis para tomar en cuenta las observaciones formuladas.

Sin duda alguna, es un trabajo extraordinario, a juzgar por el hecho de que fue poco o muy reducido lo que le dedicó al DIPr. Aunque, en realidad, su exposición gira más en torno al concepto de derecho, aprovechando el proyecto de informe de Maridakis, introduce algunos elementos de su teoria "pura". En sus observaciones presenta algunos esbozos sobre la teoría de la incorporación, afirmando que el llamado reenvío sólo es una cuestión de política legislativa, donde cada estado es libre de admitirlo o no.

En gran medida se trata de una joya prácticamente desconocida. Aunque no hay traducción al castellano de este documento (escrito en lengua francesa), presento su traducción a los interesados.

\section{ObServaciones de Hans Kelsen Al informe \\ DEL PROFESOR GEORGES MARIDAKIS ${ }^{11}$}

Mi querido colega:

$\mathrm{Su}$ excelente informe sobre "El reenvío en el derecho internacional privado" goza de toda mi consideración. Y aunque no formo parte de su comisión, trasladé al papel las reflexiones que me han inspirado algunas de las cuestiones que usted plantea, y presentan un interés teórico particular. Permitame explicarlo enseguida.

Usted escribe en la página 19: "Toda regla de derecho, por su naturaleza, contiene: un imperativo". Una regla de derecho o, lo que es lo mismo, una norma jurídica, puede tener tres funciones diferentes. El imperativo, es decir, la función de mandar, prescribir, ordenar un cierto comporta-

${ }^{11}$ La respuesta de Hans Kelsen (que no es miembro de la 23 Comisión) podrá ser utilizada por el ponente para la redacción del informe final de 20 de abril de 1957.

Nota del traductor y presentador: esta es la única nota al pie de página en el documento de Kelsen. Salvo esta, todas las notas restantes pertenecen al traductor. 
Esta revista forma parte del acervo de la Biblioteca Jurídica Virtual del Instituto de Investigaciones Jurídicas de la UNAM www.juridicas.unam.mx

http://biblio.juridicas.unam.mx

HANS KELSEN

miento humano, no es más que una de sus funciones. Un comportamiento humano es mandado jurídicamente si el comportamiento contrario es la condición de una sanción. La conducta es una acción o una omisión. Mandar, prescribir, ordenar una omisión significa: prohibir una acción.

No obstante, existen normas jurídicas que no exigen un cierto comportamiento humano, aunque lo permiten. Permitir es una función muy diferente de la de ordenar. A la orden de una autoridad jurídica-legal corresponde una obligación del sujeto; a una permisión no corresponde una obligación del sujeto. El derecho puede permitir un cierto comportamiento humano de una manera puramente negativa, en un mandato el comportamiento contrario - lo que es lo mismo- al no prohibir este comportamiento. El derecho permite un cierto comportamiento humano de una manera positiva mediante la restricción de una norma expresamente formulada, estableciendo una prohibición general, por ejemplo, el orden jurídico contiene una norma general que prohíbe la caza de ciertos animales, pero al mismo tiempo contiene una norma para la caza bajo la condición de una licencia especial.

Una tercera función es la autorización. El derecho autoriza conferir un cierto poder jurídico o una cierta autoridad jurídica a un determinado individuo. La competencia de un órgano de la comunidad jurídica es el resultado de una autorización dada por un órgano determinado mediante una norma jurídica. Por ejemplo, la Constitución permite que un órgano especial dicte normas generales, es decir, leyes; una ley autoriza a ciertos tribunales para emitir normas individuales, esto es, para que tome decisiones judiciales. La diferencia entre un permiso positivo y una autorización consiste en que la acción autorizada es un acto que crea o aplica el derecho, mientras que la acción o la omisión permitida no tiene este carácter. La función de autorización es de particular importancia, como una de las funciones esenciales del derecho como es reglamentar su propia crea- ción y su propia aplicación. 
Esta revista forma parte del acervo de la Biblioteca Jurídica Virtual del Instituto de Investigaciones Jurídicas de la UNAM www.juridicas.unam.mx

http://biblio.juridicas.unam.mx

COMENTARIOS AL PROFESOR G. MARIDAKIS

En consecuencia, la definición de Modestinus, que usted cita legis virtus haec est: imperare, vetere, permittere ${ }^{12}$, no es correcta. Vetere es un caso especial de imperare, esto significa imperare una omisión. La definición de Modestinus es demasiado restringida y no menciona una función esencial del derecho: la autorización.

Dado que, según usted, toda regla de derecho, por su propia naturaleza, contiene un imperativo, se debe concluir que las normas de derecho internacional privado siempre contienen un imperativo. Sin embargo, es muy posible que las normas de derecho internacional privado no contengan un imperativo, sino una autorización, a la que el juez no está obligado, sino solamente autorizado a aplicar a una cierta relación de derecho de otro Estado, como se suele decir, para calificar una norma de derecho internacional privado.

Usted dice luego en la página 20: la regla de derecho

tiende, en cuanto a sus fines, a la realización de la idea de derecho. Su contenido no debe contravenir el derecho; sino que debe estar al servicio del derecho. En tanto como reglas de derecho, las reglas de derecho internacional privado constituyen así mismo un imperativo, al mismo tiempo también a realizar la idea del derecho.

La expresión "el contenido de una regla de derecho no debe contravenir el derecho, debe estar al servicio del derecho", es problemática. Si una regla es una regla de derecho, nunca podrá jamás contravenir el derecho, sólo puede servir al derecho, sea cual fuese el contenido de esta regla. La expresión en cuestión no tiene sentido si la palabra "derecho" no se refiere a un derecho positivo, sino a la justicia o al derecho natural. La expresión "la regla de derecho tiende a la realización de la idea del derecho" parece justificar tal interpretación. La idea del derecho juega un papel muy importante en las conclusiones de su informe, pero lo que us-

12 Traducción: la virtud de la ley es ordenar, prohibir, permitir. 
Esta revista forma parte del acervo de la Biblioteca Jurídica Virtual del Instituto de Investigaciones Jurídicas de la UNAM www.juridicas.unam.mx

http://biblio.juridicas.unam.mx

HANS KELSEN

ted entiende por idea de derecho, no parece muy clara para mí. Usted dice: "El hombre está naturalmente inclinado al ritmo y a la armonía, por tanto para organizar una vida social armoniosa y reglada...". Si la idea del derecho es garantizar una vida social armoniosa, entonces hay que señalar que no existe una idea del derecho, sino muchas ideas del derecho; muy diferentes y contradictorias entre sí. Según algunos, sólo un orden jurídico capitalista, según otros, solo un orden jurídico comunista permite una vida social armoniosa. Más adelante, usted identifica la idea de derecho con la idea de justicia. Pero no hay una "justicia"; los hombres tienen diferentes ideas de la justicia, ideas que son muy diferentes y contradictorias. Cada derecho positivo corresponde a una de esas ideas de la justicia y, al mismo tiempo contradice otra de esas ideas. Por lo tanto, al decir que el derecho tiene por objeto la realización de la justicia, no decimos nada en absoluto; es una frase vacía, de la que no podemos obtener alguna conclusión.

En un tratado teórico sobre el derecho en general o del derecho internacional privado en particular, la hipótesis de una relación esencial entre el derecho y la justicia (o la idea del derecho) podría servir de base a un argumento si sólo existiera una idea de justicia, excluyendo la posibilidad de cualquiera otra, es decir, si hubiera una justicia absoluta. Es evidente que para una teoría científica del derecho - esto es, una teoría no metafísica ni teológica- tal idea no es aceptable. Además, usted mismo no la acepta, pues expresa cuán diferentes y contradictorias son las ideas que los legisladores toman para una justa regulación de Derecho internacional privado. Si es así, si no hay una sola idea absoluta del derecho o de la justicia, no es posible afirmar - como usted lo hace- que el legislador tiene la tarea de introducir en la regla (de derecho internacional privado) un contenido que corresponda a la idea del derecho y que los Estados tienen la obligación de "conferir a las reglas de derecho internacional privado un contenido conforme a la justicia”. 
Esta revista forma parte del acervo de la Biblioteca Jurídica Virtual del Instituto de Investigaciones Jurídicas de la UNAM www.juridicas.unam.mx

http://biblio.juridicas.unam.mx

COMENTARIOS AL PROFESOR G. MARIDAKIS

Asimismo, si hubiera una justicia absoluta, no menos cierto es que sólo el derecho internacional general podría imponer una obligación semejante a los estados, sin embargo, el derecho internacional positivo, sin duda, no. Con relación al llamado derecho internacional privado, el derecho internacional general -en su opinión - no podría imponer alguna obligación a los Estados. Usted escribe:

como no contamos en absoluto, en las circunstancias actuales con un legislador supranacional, esta tarea (de reglamentar la relación "sobre varios órdenes jurídicos", es decir, las relaciones para reglamentar el derecho internacional privado) incumbe a los legisladores de cada estado en particular (página 20).

Dado que el derecho internacional general no impone a los Estados alguna obligación relacionada con las normas del derecho nacional, conocido como derecho internacional privado, y donde no existe un derecho internacional particular, es decir, convencional, que obligue a los Estados en esta materia, los órganos legislativos de los estados tienen amplia libertad para proveer normas de derecho internacional privado, sin importar su contenido. Ni siquiera existe una necesidad jurídica de establecer en el marco de un derecho nacional normas de derecho internacional privado, es decir, de reconocer las relaciones - según una norma del derecho nacional-, las normas de otro derecho nacional deben ser aplicadas.

Además, yo no creo que sea correcto caracterizar al derecho internacional privado como una regla que estipule que en un estado ciertas relaciones sociales deban ser gobernadas por las del derecho prescrito en otro Estado, o como una norma de derecho de un Estado según la cual el derecho de otro Estado deba ser la aplicable. Esto es, que el Estado no podría aplicar su propio derecho o, en una formulación más precisa: que el órgano de un Estado no podrá aplicar las normas de su propio derecho nacional. Debido a que un individuo no es un órgano de un cierto Estado 
Esta revista forma parte del acervo de la Biblioteca Jurídica Virtual del Instituto de Investigaciones Jurídicas de la UNAM www.juridicas.unam.mx

http://biblio.juridicas.unam.mx

HANS KELSEN

ya que crea o aplica las normas del orden jurídico de su Estado. La cuestión de saber a qué orden jurídico pertenece una norma, no se relaciona con su validez básica (su fundamento). La base de la validez de todas las normas de un orden jurídico nacional es la Constitución de la comunidad formada por este orden jurídico, es decir, la Constitución del Estado cuyo derecho está en cuestión. Cuando un juez autorizado por una ley de su Estado aplica - como decimos - la norma de otro Estado, la razón de validez de la norma aplicada por el juez no es la Constitución del otro Estado, sino la Constitución de su propio Estado, al que pertenece ese órgano aplicador. Por consiguiente, el juez aplica, en este caso, una norma de su propio Estado.

Si queremos describir con precisión lo que hace el legislador de un Estado cuando promulga una regla de derecho internacional privado, hay que decir que promulga una norma cuyo contenido es idéntico al contenido de una norma de derecho de otro Estado. En lugar de decir que el derecho del Estado A permite al juez decidir la demanda de divorcio de un ciudadano del Estado B según la norma de derecho del estado $\mathrm{B}$ que reglamenta el divorcio, sería más correcto decir que una ley del Estado A autoriza a un juez para decidir la demanda de divorcio de un ciudadano del Estado B como norma del derecho del estado A, es decir, su propio estado, norma cuyo contenido es idéntico al contenido de una norma de derecho del Estado B reglamentando el divorcio. La ley del Estado A no determina directamente el contenido de la norma a ser aplicada por los tribunales; sólo determina el Estado a cuyo derecho es necesario remitirse, o sea, el del Estado B, y autoriza al tribunal a dar una norma que debe aplicarse con un contenido idéntico al contenido de la norma de derecho del estado $\mathrm{B}$ que regula el divorcio. Aunque, de acuerdo con la teoría tradicional, usted dice que en virtud de la regla de derecho internacional privado de un cierto Estado, que es "el derecho de otro Estado" el que debe aplicar, no obstante, lo que dice no significa que "el Estado exprese su voluntad de "no aplicar su propia 
Esta revista forma parte del acervo de la Biblioteca Jurídica Virtual del Instituto de Investigaciones Jurídicas de la UNAM www.juridicas.unam.mx

http://biblio.juridicas.unam.mx

COMENTARIOS AL PROFESOR G. MARIDAKIS

legislación", que no se debe a la voluntad del Estado de no aplicar su propia ley...”. Esta afirmación es correcta, pero no es el derecho de otro estado, sino su propio derecho, el que aplica el Estado. Sin embargo, en lugar de llegar a este resultado, usted dice que una regla de derecho internacional privado, significa simplemente que el Estado cumple con su obligación de "conferir a sus reglas de derecho internacional privado un contenido conforme a la justicia" (página 28). Ya dejé explicado por qué esta fórmula no me parece aceptable.

La cuestión principal que trata su informe es si la regla de derecho internacional privado de un cierto Estado puede o debe referirse únicamente al derecho sustantivo en vigor en otro Estado o si se puede o debe referirse también al derecho internacional privado de ese otro Estado. Desde el punto de vista del positivismo jurídico, no cabe duda que una legislatura puede estipular una u otra. La cuestión de saber cuál de estas dos opciones es preferible es una cuestión de política jurídica (rechtspolitik); y desde este punto de vista las dos soluciones son posibles. Usted le da preferencia a la primera solución, de hecho, cree que las reglas de derecho internacional privado de un cierto Estado deben prescribir únicamente el derecho sustantivo o material de otro estado y no su derecho internacional privado. Es probable que pueda justificar esta opinión con argumentos basados en algunos juicios de valor. Pero no podemos, como usted lo hace, ya que excluye la otra posibilidad, es decir, excluye que la legislatura de un cierto Estado prescriba que sus órganos judiciales también puedan tomar en cuenta el derecho internacional privado en el otro Estado. Usted afirma que ello es "gobernado por la fuerza de las cosas," que es "inconcebible y contrario a la naturaleza de las cosas." Esto presupone, sin embargo, que es justo, en un sentido absoluto, la regla según la cual el tribunal sólo debe considerar el derecho sustantivo de otro Estado, con exclusión del derecho internacional privado. Y aquí es donde está su pensamiento, ya que usted escribe que un estado, al pres- 
Esta revista forma parte del acervo de la Biblioteca Jurídica Virtual del Instituto de Investigaciones Jurídicas de la UNAM www.juridicas.unam.mx

http://biblio.juridicas.unam.mx

HANS KELSEN

cribir una regla de derecho internacional privado aplica una norma de derecho sustantivo de otro estado, otorgando a su norma de derecho internacional privado "un contenido conforme a la justicia" y que "sería inconcebible y contrario a la naturaleza de las cosas" —es decir, contrario a la justicia-

que un Estado prescriba a sus órganos tomar en cuenta para una cierta relación el derecho extranjero para que la justicia sea una realidad, y que a la vez se acepte simultáneamente el derecho internacional privado establecido en ese otro Estado. Ya que, después de haber formulado su voluntad en cuanto al país de la legislación que debe ser aplicada para que la justicia sea un hecho, el Estado se contradiga ordenando a sus órganos buscar, con este mismo fin, la legislación de otro país (página 23).

Que el Estado "se contradiga" significa que una prescripción de su parte de tomar en cuenta también el derecho internacional privado del otro Estado seria contraria a la justicia. Pero aquí se le olvida que, según su propia teoría, el otro estado también confiere a sus reglas de derecho internacional privado "el contenido acorde a su justicia" y que, por lo tanto, un Estado que prescribe tomar en cuenta el derecho internacional de otro Estado, no contradice la "justicia"; él ordena solamente considerar la justicia del otro Estado, aunque esta justicia puede ser diferente a su propia justicia. Ignora el hecho de que la justicia no es una ni absoluta, sino que, como usted mismo lo admite, las concepciones de justicia son muy diferentes. Es muy posible que el legislador de un cierto Estado considere que la aplicación a una cierta relación de derecho sustantivo de otro estado sea consistente conforme a la justicia sólo si también se tienen en cuenta el derecho internacional privado de ese Estado. Si se admite que no hay justicia absoluta, no puede alegarse una presunta incompatibilidad con la idea de justicia para excluir la posibilidad de establecer, en una norma de derecho internacional privado, se tome en cuenta 
Esta revista forma parte del acervo de la Biblioteca Jurídica Virtual del Instituto de Investigaciones Jurídicas de la UNAM www.juridicas.unam.mx

http://biblio.juridicas.unam.mx

COMENTARIOS AL PROFESOR G. MARIDAKIS

no sólo la norma del derecho material, sino también del derecho internacional privado de otro estado.

Para "arreglar el mal estado de las cosas producidas por la división de los estados en dos grupos, donde uno admite como punto de vinculación el domicilio (residencia habitual) y el otro la nacionalidad," usted recomienda en la página 46 encontrar el remedio "únicamente en la naturaleza de las reglas del derecho".

Usted caracterizaria esta "naturaleza" diciendo que la regla de derecho es, en general, formulada tomando en cuenta un término medio $\mathrm{y}$

formulada en lo abstracto, en vista de lo plerumque quod fit, ${ }^{13}$ es raro que la regla de derecho cubra exactamente la relación considerada. Por lo general, excede en un caso y en otro es insuficiente (páginas 46/7).

La regla abstracta (i.e., general) pequeña, por lo tanto, sea considerada como demasiado amplia o demasiado estrecha, lo que quiere decir que es "insuficiente" en ambos casos, que su aplicación estricta a un caso concreto puede conducir a un resultado que "no es apropiado para la ocasión de que se trata". Que el resultado no es apropiado sólo puede significar que la decisión del órgano que aplica la regla general a un caso concreto no es justa.

Sin embargo, es cierto que, como usted escribe, "las reglas del derecho en general son formuladas en consideración... de lo que ocurre más comúnmente", ciertamente no se puede decir que la regla de derecho (de ordinario), supera la relación de que se trata, o es insuficiente. La falta de una regla abstracta no puede ser otra que la excepción. Pero el punto crucial es que cuando decimos que una regla general es insuficiente, la aplicación estricta de esta regla a un caso concreto conduce a un resultado que no es adecuado a las circunstancias consideradas en el caso concreto, que la decisión del órgano que aplica estrictamente la regla

13 Traducción: sucede comúnmente. 
Esta revista forma parte del acervo de la Biblioteca Jurídica Virtual del Instituto de Investigaciones Jurídicas de la UNAM www.juridicas.unam.mx

http://biblio.juridicas.unam.mx

HANS KELSEN

abstracta a un caso concreto, no es justa. Cuando decimos que un juicio de valor es expresado en forma subjetiva, normalmente, la aplicación estricta de la regla abstracta es considerada como apropiada por la parte en cuyo favor se aplicó, y como inapropiada por la otra parte. Es imposible formular una norma jurídica - general o individual, abstracta o concreta- que no pueda considerar un punto de vista u otro, como inapropiado o injusto. Continuando con su análisis de la "naturaleza de las reglas del derecho", sin embargo usted escribe:

por tanto: es necesario que el derecho formulado en un término medio en una regla abstracta se transforme en derecho "exacto"... apropiado a la circunstancia considerada. Este es el juez en el que el legislador confía la tarea de aplicar la regla abstracta al caso concreto, para adaptarla a las dimensiones del mismo con el fin de atribuir "con exactitud a cada uno lo que le conviene." El juez... convertirá el derecho formulado en una regla abstracta en el derecho requerido por las circunstancias particulares (página 47).

Esto, no me parece exacto. Hay, en efecto, leyes que confieren a un juez el poder que usted designa como la regla de transformación abstracta en derecho exacto, pero también hay leyes que no confieren tal poder. Transformar la regla abstracta en derecho "exacto" significa transformar un derecho injusto en derecho absolutamente justo, lo cual es imposible. Lo que el legislador puede hacer - pero no necesariamente lo hace- es autorizar al juez a no aplicar la norma general establecida por el legislador, si pudiera considerar la aplicación como inadecuada, es decir, injusta, y autorizar que aplique la norma que considere apropiada. Esto significa que el legislador autoriza al juez a conformar su decisión a su idea de hacer justicia, es decir, lo autoriza para actuar como legislador. Si esta autorización es el remedio buscado para resolver el estado de cosas malsanas encontradas, entonces no podemos decir que se trata de un remedio que se encuentra "en la naturaleza de las reglas 
Esta revista forma parte del acervo de la Biblioteca Jurídica Virtual del Instituto de Investigaciones Jurídicas de la UNAM www.juridicas.unam.mx

http://biblio.juridicas.unam.mx

COMENTARIOS AL PROFESOR G. MARIDAKIS

del derecho", ya que existen reglas de derecho que no contienen tal autorización; y si el juez está autorizado para crear una norma conforme a idea subjetiva de la justicia, no podemos decir que se trata de una norma requerida por las circunstancias, ya que no precisamente - como esta fórmula tomada de la teoría del derecho natural lo indicaparticipa en las circunstancias, pero requiere de la idea subjetiva de la justicia que hace el juez. La cuestión de si es recomendable conferirle al juez el poder de actuar como legislador es una cuestión de política jurídica (rechtspolitik). La respuesta depende de la elección entre dos valores o dos principios de justicia: el principio de seguridad jurídica (rechtssicherheit), que no puede alcanzarse si se desconocen ciertas particularidades de los casos concretos, y el principio de flexibilidad del derecho, que demanda tener en cuenta siempre que sea posible todas las circunstancias particulares de los casos concretos.

En lo que respecta al derecho internacional privado, usted prefiere el principio de flexibilidad. Usted dice en la página 49:

Si la estricta aplicación de la regla (de derecho internacional privado que prescribe sea aplicada a ciertas relaciones el derecho de otro Estado) conduce a una injusticia, debe ser accesible al juez apartarse de la regla y designar la ley de otro Estado, cuya aplicación se impone en razón de circunstancias especiales.

Bien podría hacer tal recomendación, pero no en nombre de la "justicia", es decir, en nombre de la justicia absoluta. No se puede hacer en nombre de una justicia relativa, lo que no excluye la posibilidad de recomendar - en nombre de una justicia que no es menos justicia que la primera, es decir, a nombre de la justicia de la seguridad jurídica- no conferirle al juez el poder de apartarse de la ley, sino de obligar al contrario a la aplicación estricta.

Le ruego acepte, mi estimado colega, la expresión de mi más alta consideración y mis dedicados sentimientos. 
Esta revista forma parte del acervo de la Biblioteca Jurídica Virtual del Instituto de Investigaciones Jurídicas de la UNAM

III. BIBLIOGRAFÍA

SÁNCHEZ LORENZO, Sixto, "Estado democrático, posmodernismo y el derecho internacional privado", Revista de Estudios Jurídicos, segunda época, núm. 10, Universidad de Jaen, 2010.

SILVA, Jorge Alberto, Aplicación de normas conflictuales. La aportación del juez, México, UACJ-Fontamara. 2010.

VITTA, Edoardo, Cours générale de droit internationale prive, vol. 126, 1979-1. 